\title{
Oxidative Stress Measurement and Prediction of Epileptic Seizure in Children and Adults With Severe Motor and Intellectual Disabilities
}

\author{
Masahito Morimoto $^{\mathrm{a}, \mathrm{b}, \mathrm{c}}$, Shigeko Satomura ${ }^{\mathrm{b}}$, Toshiaki Hashimoto ${ }^{\mathrm{b}}$, Etsuro Ito $^{\mathrm{a}}$, Shojiro Kyotani ${ }^{\mathrm{a}}$
}

\begin{abstract}
Background: The medical care of severe motor and intellectual disabilities (SMID) depends on the empirical medical care. Epileptic seizure specific to SMID is difficult to suppress using anti-epileptic drugs, and its tendency to persist for long periods poses an issue. The present study was undertaken to evaluate the relationship between epileptic seizure in cases with SMID and oxidative stress in the living body by examining endogenous antioxidants, the degree of oxidation (reactive oxygen metabolites (d-ROMs)), and the biological antioxidant potential (BAP) as indicators.
\end{abstract}

Methods: Target patients were 43 SMID epilepsy patients. Blood was sampled before breakfast and medication. As for the specimen, d-ROMs and BAP were measured using the free radical analyzer.

Results: The present study did not reveal any correlation between endogenous antioxidants (albumin) and the frequency of epileptic seizures. On the other hand, d-ROMs were correlated with the frequency of epileptic seizure. In particular, strong correlations between the frequency of epileptic seizures and the d-ROMs/BAP ratio as well as the $\mathrm{BAP} / \mathrm{d}-\mathrm{ROMs}$ ratio were noted.

Conclusions: These results indicate that the use of d-ROMs and BAP as biomarkers can provide a tool for predicting the prognosis of epileptic seizures in patients with SMID.

Keywords: Motor and intellectual disabilities; Epilepsy; Seizure; Oxidative stress; d-ROMs test; Biomarker

\section{Introduction}

The purpose of this study was to clarify it about influence of

Manuscript accepted for publication March 22, 2016

${ }^{a}$ Tokushima Bunri University, Graduate School of Pharmaceutical Sciences, Tokushima, Japan

bJapanese Red Cross Tokushima Hinomine Rehabilitation Center for People With Disabilities, Tokushima, Japan

${ }^{\mathrm{c} C o r r e s p o n d i n g ~ A u t h o r: ~ M a s a h i t o ~ M o r i m o t o, ~ T o k u s h i m a ~ B u n r i ~ U n i v e r s i t y, ~}$ Graduate School of Pharmaceutical Sciences, Nishihama, Yamashiro-cho, Tokushima 770-8514, Japan. Email: morimoto@hinomine-mrc.jp

doi: http://dx.doi.org/10.14740/jocmr2534w the oxidation stress to give to a seizure of severe motor and intellectual disabilities (SMID). Furthermore, exploring the oxidation stress measurement is the clinical possibility for the SMID epilepsy prediction.

The elevation of oxidative stress in response to the excessive expression of reactive oxygen species and a reduction in the antioxidant potential are reportedly associated with not only the promotion of aging, but also various diseases including hypertension, diabetes mellitus, and rheumatoid arthritis [1-3].

SMID is "patient having severe mental disabilities and severe limbs disorder". SMID is often complicated by epilepsy, which tends to be intractable [4]. The complete control of epileptic seizures in such cases is difficult, even with a combination of multiple antiepileptic drugs (AEDs). Furthermore, the underlying cause of persistent epileptic seizures in cases with SMID remains unexplained. In patients with epilepsy, the excessive expression of free radicals, leading to encephalopathy, has recently attracted close attention $[5,6]$. With these reports in mind, we recently explored the factors that are involved in epileptic seizures in patients with SMID, paying close attention to oxidative stress. Using endogenous antioxidants (albumin), the degree of oxidation (reactive oxygen metabolites (d-ROMs)), and the biological antioxidant potential (BAP) as potential predictors of epileptic seizure, we analyzed their actual associations with epileptic seizure.

\section{Patients and Methods}

\section{Patients}

The present study involved 43 patients with SMID complicated by epilepsy who stayed at the Japanese Red Cross Tokushima Hinomine Rehabilitation Center for People with Disabilities during a 1-year period from January 1, 2015 to December 31, 2015.

\section{Background variables}

The following background variables of the individual patients were investigated using information contained in their medical records: 1) basic information (age, sex, and main diagnosis), and 2) mean frequency of epileptic seizures (mean frequency during a 1-month period between January 1, 2015 to December 31, 2015). 
Table 1. The Measurement Evaluation Standard Value of $d$ ROMs Test and BAP Test

\begin{tabular}{|ll|}
\hline Evaluation & Standard value \\
\hline d-ROMs test & \\
\hline Normal range & $250-300$ U.CARR* \\
Border range & $301-320$ U.CARR \\
\hline Low-level oxidative stress & $321-340$ U.CARR \\
Intermediate-level oxidative stress & $341-400 \mathrm{U} . \mathrm{CARR}$ \\
\hline High-level oxidative stress & $401-500 \mathrm{U} . \mathrm{CARR}$ \\
\hline Very high-level oxidative stress & $>500 \mathrm{U} . \mathrm{CARR}$ \\
\hline BAP test & \\
\hline Normal range & $>2,200 \mu \mathrm{mol} / \mathrm{L}$ \\
\hline Border range & $2,000-2,200 \mu \mathrm{mol} / \mathrm{L}$ \\
\hline Slight lack state & $1,800-1,999 \mu \mathrm{mol} / \mathrm{L}$ \\
Lack state & $1,600-1,799 \mu \mathrm{mol} / \mathrm{L}$ \\
\hline Severe lack state & $1,400-1,599 \mu \mathrm{mol} / \mathrm{L}$ \\
Very severe lack state & $<1,400 \mu \mathrm{mol} / \mathrm{L}$ \\
\hline
\end{tabular}

\section{Parameters measured}

Blood samples were SMID in a state without poor physical condition and an epilepsy attack. Blood was sampled before breakfast and before medication $(6: 00 \mathrm{am})$; the samples were then centrifuged at 3,000 rpm for 10 min using a tabletop centrifuge (model 2410; Kubota Corporation, Tokyo) to isolate the plasma. The resulting plasma samples were then used to measure the following parameters.

\section{Biochemistry}

The albumin and uric acid levels were measured using LAboSPECT006 (HITACHI, Tokyo) and the reagents Cica Liquid ALB and Cica Liquid UA (Kanto Chemical, Tokyo).

\section{Indicators of oxidative stress (d-ROMs test)}

Indicators of oxidative stress were measured using the free radical analyzer FREE CARRIO DUO (Diacron International, Italy). Each plasma sample $(20 \mu \mathrm{L})$ was placed in a cuvette filled with a buffer ( $\mathrm{pH} 4.8)$. The cuvette was then placed upside down and agitated (to induce the release of $\mathrm{Fe}^{2+}$ and $\mathrm{Fe}^{3+}$ from the blood proteins). $\mathrm{Fe}^{2+}$ and $\mathrm{Fe}^{3+}$ serve as catalyzers, resulting in the degradation of blood hydroperoxide into an alkoxy radical and a peroxy radical. Then, a color-developing chromogen (N,N-diethyl-p-phenylenediamine) was placed in a volume of $20 \mu \mathrm{L}$, resulting in the oxidation of the chromogen substrate by the free radicals to yield a red-colored radical cation. The cuvette was once again turned upside down and agitated, then placed in a photometer for optical measurement. U.CARR is used for the unit, and 1 U.CARR is equivalent to

\section{a d-ROMs test value}

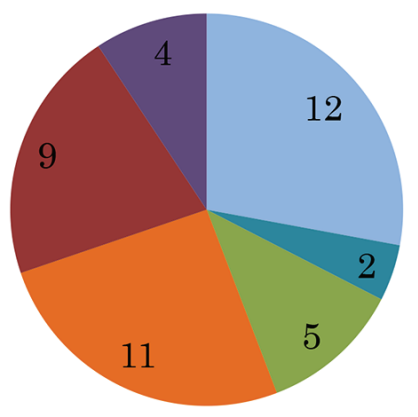

Normal range

Border range

- Low-level

Intermediate level

- High-level

- Very high-level

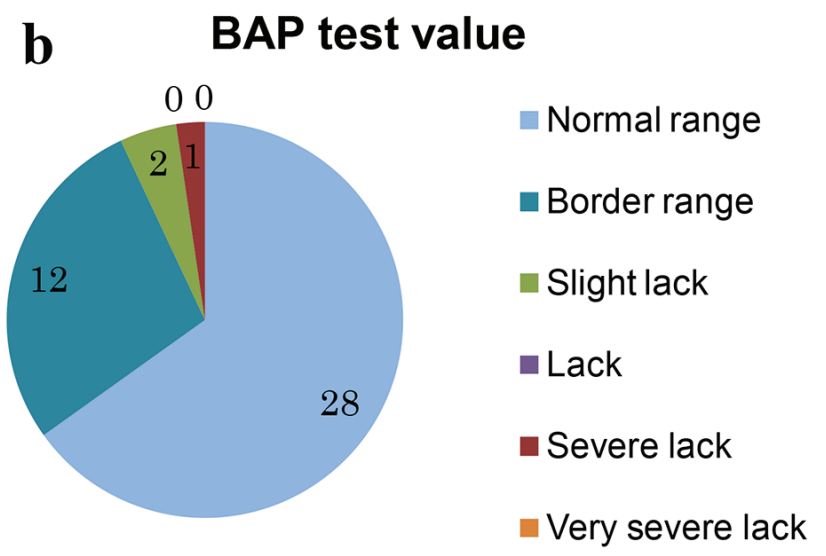

Figure 1. Result of a measurement of d-ROMs test (a) and BAP test (b).

the $\mathrm{H}_{2} \mathrm{O}_{2}$ of $0.08 \mathrm{mg} / \mathrm{dL}$.

\section{Indicators of antioxidant potential (BAP test)}

Indicators of BAP were measured using the free radical analyzer FREE CARRIO DUO (Diacron International, Italy). A chromogen for BAP (a reagent containing trivalent iron; 50 $\mu \mathrm{L}$ ) was added to a cuvette to induce red coloration. The cuvette was placed upside down, and the color-developing concentration was measured using a photometer. Then, each plasma sample $(10 \mu \mathrm{L})$ was placed into a cuvette and agitated. The cuvette was then placed in a thermostat for $5 \mathrm{~min}$. Then, the cuvette was placed into a photometer for optical measurement ( $\mu \mathrm{mol} / \mathrm{L}$ is used for the unit).

\section{Degree of oxidative stress (d-ROMs value/BAP value)}

To determine the oxidative stress at the time of measurement, the d-ROMs value was divided by the BAP value. This ratio of the free radical level to the antioxidant potential was named "degree of oxidative stress." 


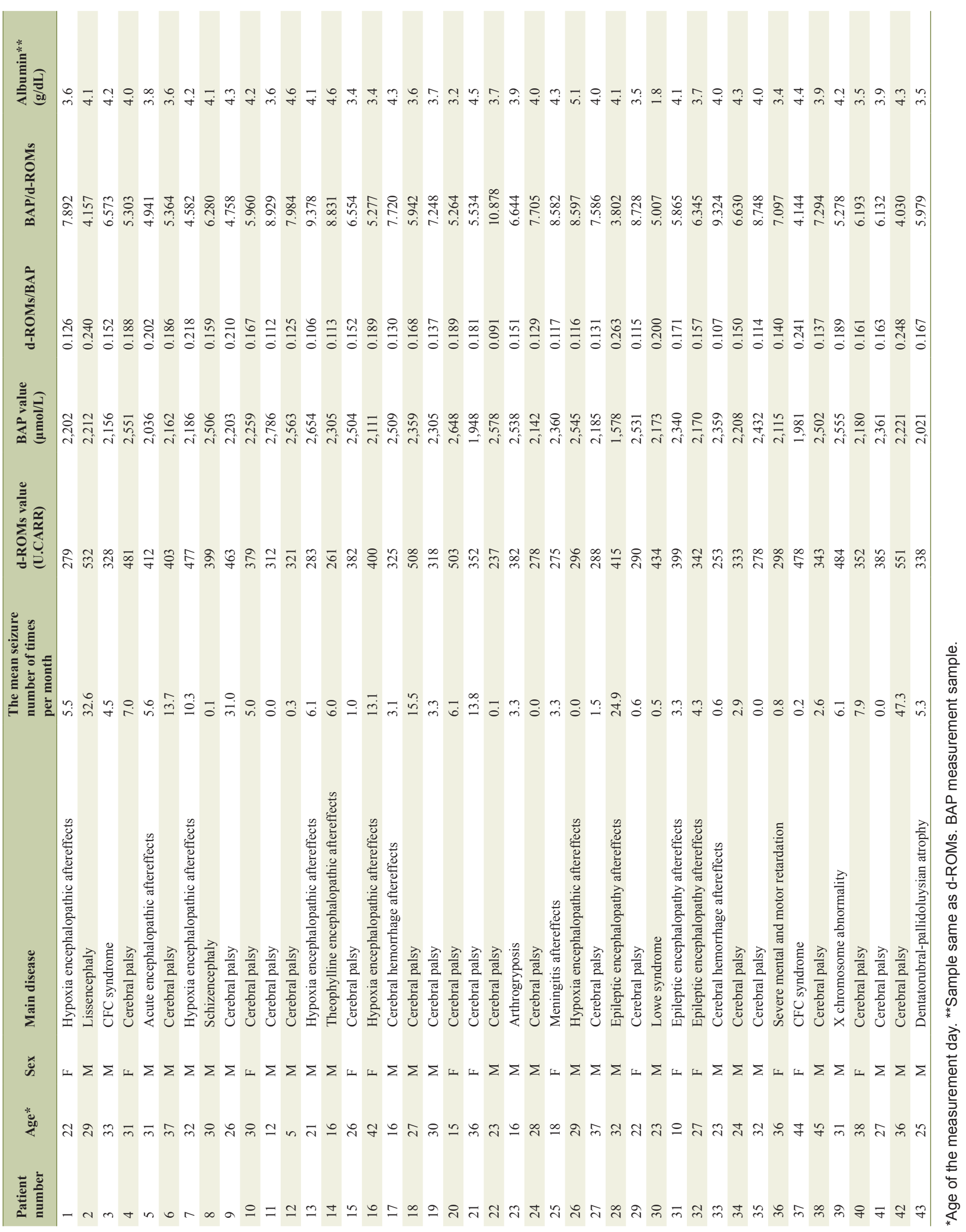



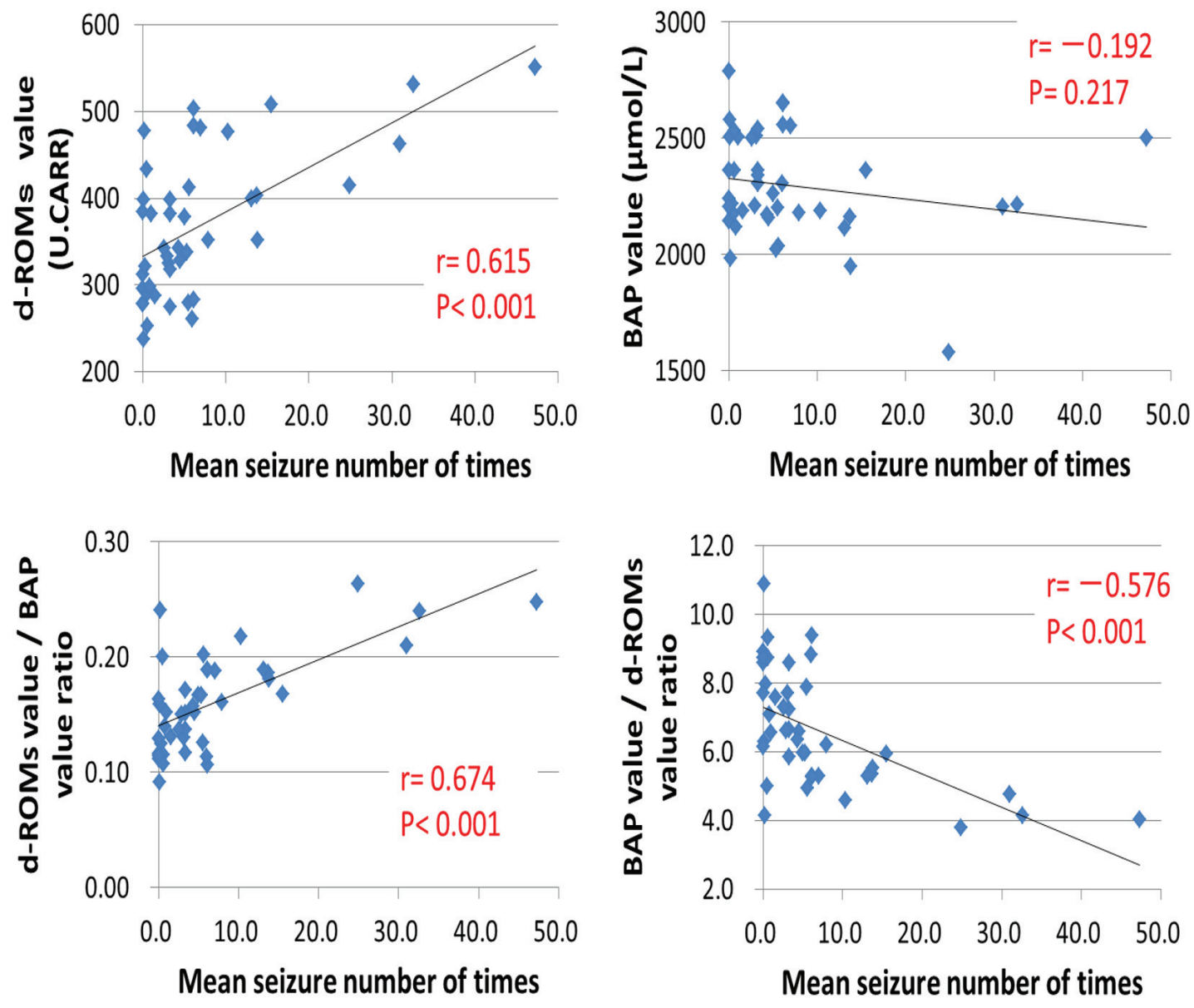

Figure 2. Relations of the mean seizure number of times and each measurement.

Antioxidant potential (BAP valueld-ROMs value)

To assess the antioxidation capability of the measured BAP towards free radicals, the BAP value was divided by the dROMs value. This ratio of BAP to the free radical level was named "antioxidation capability".

\section{Evaluation of measured data}

The criteria given in Table 1 were used for the evaluation. Normal value is d-ROMs value $<320 \mathrm{U}$.CARR, and BAP value $>2,000 \mu \mathrm{mol} / \mathrm{L}$. Other values assumed it an abnormal value.

\section{Statistical analysis}

The statistical analysis software used IBM SPSS Statistics version 21 and Microsoft Excel statistics. Chi-square test (relation of sex, main diagnosis, d-ROMs value, and BAP value) and regression line (relation of age, epilepsy seizure number of times, albumin, "degree of oxidative stress", and "antioxidation capability") were used. The significance level was set at
$5 \%$ (two-tailed). In all the tests, $\mathrm{P}<0.05$ was regarded as being statistically significant.

\section{Ethical consideration}

This study was approved in advance by the Ethics Committee of the Japanese Red Cross Tokushima Hinomine Rehabilitation Center for People with Disabilities. Because patients with SMID have difficulty expressing their intentions, informed consent was obtained in writing from their family members. The investigation used the medical records of individual patients. The data collected were anonymized to avoid identification of the individuals and were managed and analyzed using computers that were not networked.

\section{Results}

Background variables are shown in Table 2.

The age of the patients was $27.3 \pm 8.9$ years (mean $\pm \mathrm{SD}$ ). There were 29 males and 14 females. The most common major diagnosis was cerebral palsy (21 cases), followed by hypoxia 

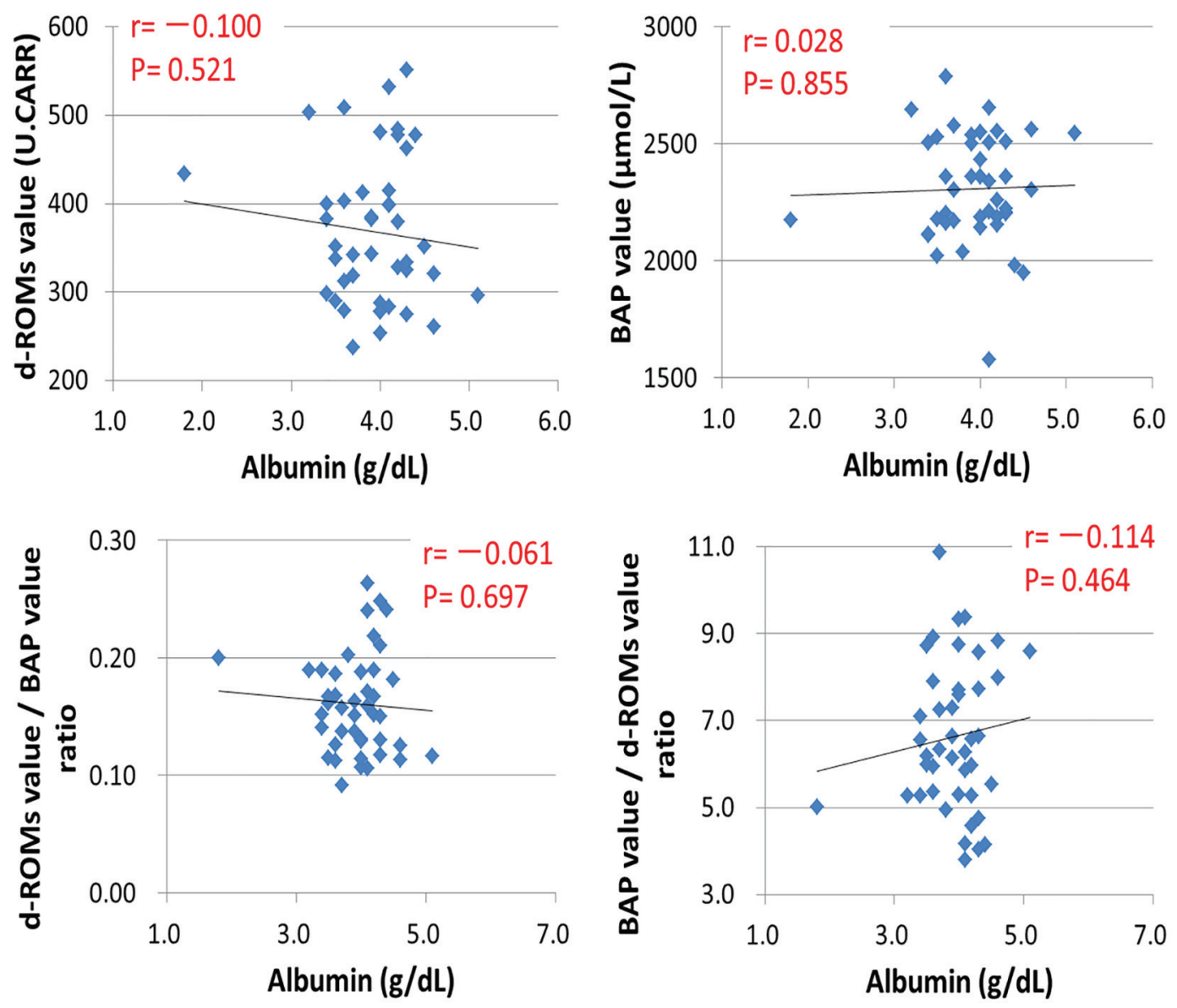

Figure 3. Relations of albumin and each measurement.

encephalopathic aftereffects, epilepsy encephalopathic aftereffects, etc.

The mean monthly frequency of seizures was $7.0 \pm 10.0$ times (mean $\pm \mathrm{SD}$ ), with a maximum of 47.3 , a minimum of 0.0 , and a median of 3.3 .

Figure 1 shows the results of the d-ROMs test and the BAP test. In the d-ROMs test, abnormal oxidative stress was noted in more than $50 \%$ of the cases. In the BAP test, on the other hand, only $7.0 \%$ of the cases were abnormal.

It was analyzed about relation with background variables and d-ROMs value and BAP value.

Sex: $\mathrm{d}-\mathrm{ROMs}$ value, $\mathrm{P}=0.967$, BAP value, $\mathrm{P}=0.503$ (Chi-square test); age: $\mathrm{d}-\mathrm{ROMs}$ value, $\mathrm{P}=0.115$, $\mathrm{BAP}$ value, $\mathrm{P}=0.001$ (regression line); main disease: $\mathrm{d}-\mathrm{ROMs}$ value, $\mathrm{P}=$ 0.274 , BAP value, $\mathrm{P}=1.000$ (Chi-square test).

In age and BAP value, correlation was accepted. BAP value decreased as age became higher.

\section{Relation with mean of epileptic seizures}

Results of the analysis are shown in Figure 2. Each parameter was correlated with the mean frequency of seizures (BAP is excluded). When the epilepsy attack number of times in- creased, the quantity of free radical increased, but the antioxidant power was kept. The oxidative stress also increased, while the antioxidation capability decreased.

\section{Relation with albumin}

Results of the analysis are shown in Figure 3. None of the parameters were correlated with the albumin level.

\section{Association between d-ROMs value and BAP value}

Results of the analysis are shown in Figure 4. The d-ROMs value and the BAP value were not correlated. Even when the free radical level increased, the antioxidant potential tended to be preserved.

\section{Association between d-ROMs value/BAP value ratio and BAP value/d-ROMs value ratio}

Results of the analysis are shown in Figure 5. There was a strong correlation between the d-ROMs value/BAP value ratio 


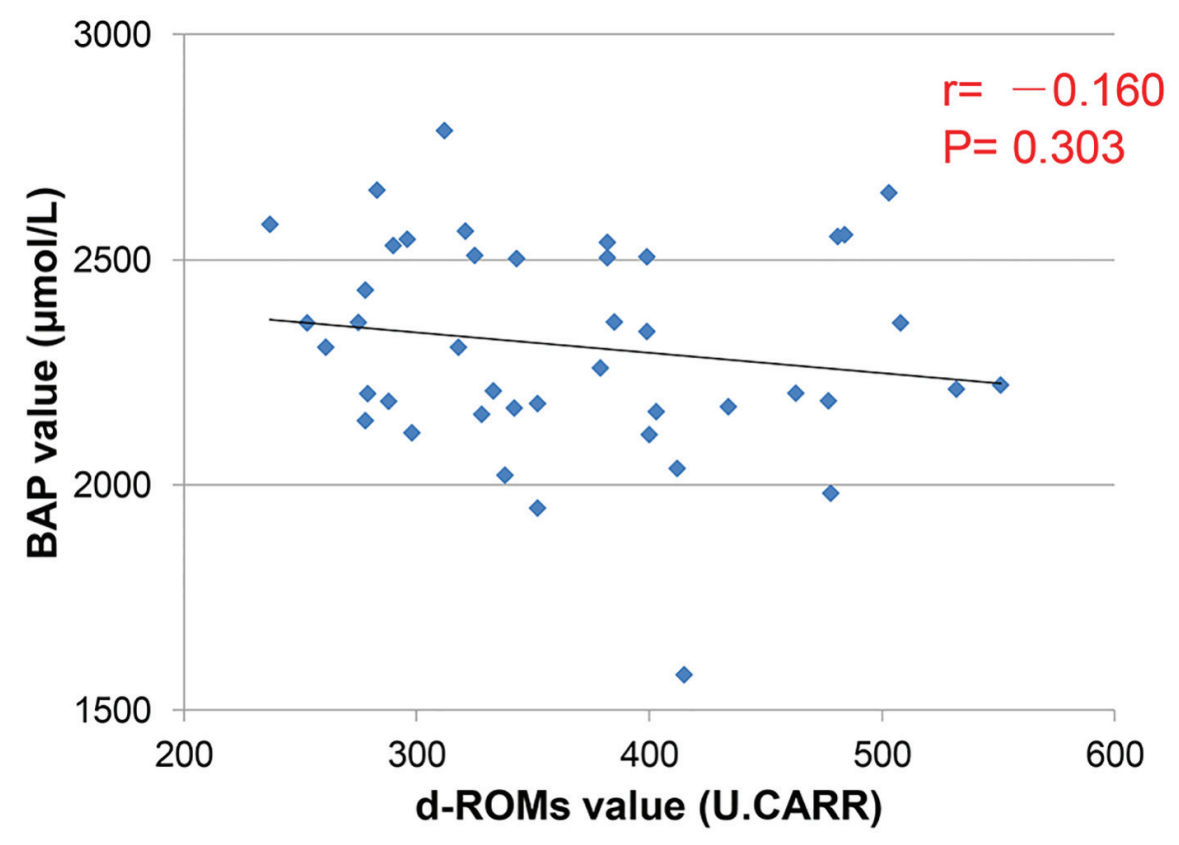

Figure 4. Relation between d-ROMs value and BAP value.

and the BAP value/d-ROMs value ratio. As the oxidative stress increased, the antioxidation capability decreased.

\section{Discussion}

Correlation between background variables and d-ROMs value and BAP value is analyzed. As a result, there was accepted negative correlation only in age and BAP value. It is generally known that oxidation stress load grows with aging. It is supposed that SMID epilepsy patient is the same. However, as for the measurements, $40 / 43(93.0 \%)$ were within the normal range. There is the tendency that antioxidant power decreases by aging. But a patient of the abnormal values has very little. "Age" is thought not to be a factor influencing this study.

An open issue in the management of intractable epilepsy

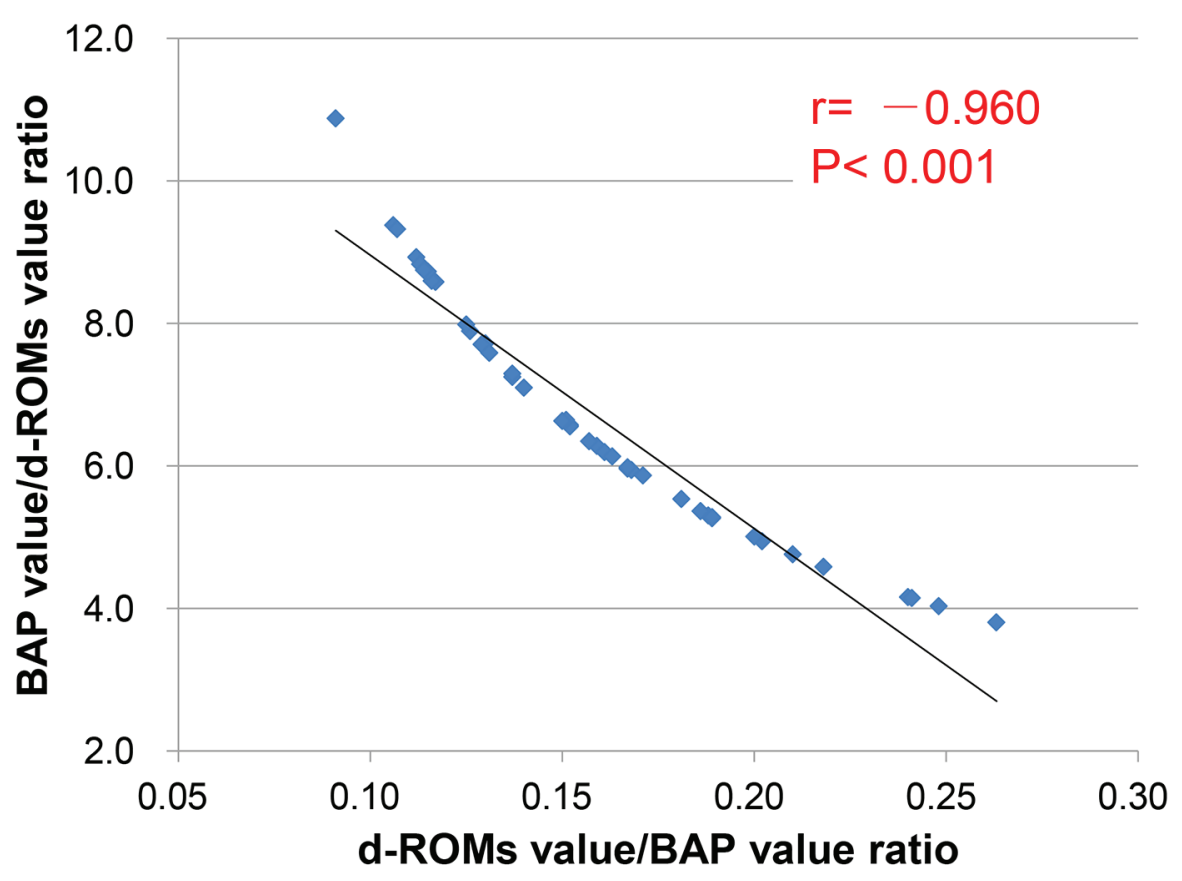

Figure 5. Relation between d-ROMs value/BAP value ratio and BAP value/d-ROMs value ratio. 
associated with SMID is how to reduce the frequency of seizures. Clarifying the mechanism responsible for the onset of seizures and predicting their occurrence in individual cases are the most important issues in the treatment of this condition. In past studies, epilepsy has been considered to be associated with excessive oxidative stress. Epilepsy seen in patients with SMID also seems to involve oxidative stress, but no detailed studies have been conducted.

In the present study, the influence of oxidative stress on epileptic seizures associated with SMID was evaluated. In this study, patients with SMID who developed epilepsy were found to have elevated oxidative stress levels following epileptic seizures, as is known to occur in epileptic patients in general [7]. Furthermore, a higher frequency of seizures was associated with an elevated free radical level and a tendency toward a reduced oxidative potential. These findings suggest that epilepsy seen in patients with SMID is affected by oxidative stress, as in other previously reported diseases. Because patients with SMID do not undergo normal brain development, the oxidative stress level was estimated to be relatively high regardless of seizure control. This view was not supported in all the cases observed in this study, and the oxidative stress level was found to depend on seizure control, as shown in Figure 2.

Imazato et al reported a correlation between antioxidation (BAP) and endogenous antioxidants (albumin and uric acid) in patients with rheumatoid arthritis [8]. In the present study, however, albumin level was not correlated with the BAP value. This finding suggests that as in the frail patients who tend to show low albumin level [9], SMID patients tend to be poorly nourished because nutrients are often obtained from tube feeding or central vein alimentation, unlike in healthy individuals, resulting in low albumin level, i.e., the tendency of SMID patients being skinny may be the cause of the low albumin level.

As the evidence, it is reported in body mass index (BMI) standard that SMID is significantly much lean constitution [10]. It is guessed that an albumin low value influences relations with BAP.

In lifestyle-related disease patient and puerpera, the change in the extent of oxidation (d-ROMs value) is associated with the change in antioxidant potential (BAP value) [11, 12]. In patients with epilepsy associated with SMID, on the other hand, the present study results suggest that the d-ROMs value and the BAP value are independent of each other. We may therefore estimate that the preservation of the BAP value is affected by factors other than endogenous antioxidants. The identification of such factors is an open question. One possible hypothesis is that the carnitine level in vivo may affect the BAP value. This hypothesis is based on the following findings: 1) patients with SMID-associated epilepsy often receive massive treatment with the AEDs valproate sodium (VPA) or carnitine replacement therapy for the correction of hypocarnitinemia caused by tube feeding $[13,14]$; 2) carnitine is also known to serve as an antioxidant; and 3) the increased expressions of SOD, catalase, and glutathione peroxidase, etc., following carnitine treatment have been reported [15]. So, carnitine appears to be closely involved in the preservation of the antioxidant potential, as seen in this study. Including the carnitine measurement, we want to assume the influence of the exogenous antioxidant material in SMID epilepsy a problem in future.
A strong correlation was noted between the d-ROMs value/BAP value ratio (oxidative stress) and the BAP value/dROMs value ratio (antioxidant potential). In this connection, Ueda et al [4] reported that the age at the time of the first onset of SMID-associated epilepsy was $2.7 \pm 4.1$ years (mean \pm SD) and that cases with an age of first onset of less than 1 year were often intractable. Also, the patients with SMID who were included in the present study had a relatively long duration of epilepsy that was intractable, if we consider the age at the time of the first onset of epilepsy. So, these patients may have cumulative damage to brain/nerve function and relatively high free radical levels. Furthermore, a reduction in antioxidation capability in response to elevated levels of oxidative stress arising from long-term, persistent seizures, rather than exposure to temporary oxidative stress, is likely to be inevitable.

In this study, each ratio was evaluated using the criteria for assessing the d-ROMs value. Degree of oxidative stress, as defined by the d-ROMs value/BAP value ratio, d-ROMs value showed an abnormal value at 26/28 (92.9\%) if more than 0.15 . Antioxidation capability, defined by the BAP value/d-ROMs value ratio, d-ROMs value showed an abnormal value at $26 / 28$ (92.9\%). d-ROMs value showed normal level at 15/17 (88.2\%) if less than 7.0. Degree of oxidative stress more than 0.15 , antioxidation capability is less than 7.0. It is thought with a standard rate of the oxidation condition of stress of SMID epilepsy.

Further follow-up is needed to determine whether these ratios are applicable to all cases of SMID-associated epilepsy and whether they can be used to prevent oxidative stress-associated disease and to adjust AED dosages by predicting the frequency of epileptic seizure. In any event, these parameters are likely to become useful biomarkers of SMID-associated epilepsy. Furthermore, we can measure d-ROMs test and BAP test with a very small amount of blood $(30 \mu \mathrm{L})$ of the fingertip. This clinical inspection is a simple, easy, quickly, cheap, low aggression. We think with the oxidation stress rating system that it is easy to use for clinical practice.

\section{Conclusions}

No evidence-based medicine (EBM) is applicable to the management of SMID patients, and the management of these patients presently relies on empiric care. SMID cannot do intention expression by oneself. Under such circumstances, epileptic seizures sometimes persist for long periods, and the early detection and treatment of diseases that are known to have an increasing incidence with aging (e.g., cancer and lifestylerelated chronic disease) is sometimes difficult. Therefore, we were looking for a useful objective index. The present study suggests that screening using the d-ROMs value or the BAP value may be useful as an objective indicator of SMID, for which little subjective information is available. Furthermore, these parameters may serve as biomarkers for preventing the onset of oxidative stress-related disease and for adjusting AED dosages. Establishing EBM will be important for the treatment of SMID and for providing more uniform medical care and drug therapy, with fewer discrepancies among healthcare providers. 


\section{References}

1. Digiesi V, Oliviero C, Gianno V, Rossetti M, Fiorillo C, Oradei A, Lenuzza $\mathrm{M}$, et al. [Reactive metabolites of oxygen, lipid peroxidation, total antioxidant capacity and vitamin $\mathrm{E}$ in essential arterial hypertension]. Clin Ter. 1997;148(11):515-519.

2. Ikebuchi M, Nishino Y, Maegawa H, Kashiwagi A. Effect of hyperglycemia on oxidative stress and antioxidant potential in patients with type 2 diabetes. Diabetology International. 2010;1(2):72-77.

3. Nonaka T, Fukuda K, Hamanishi C. Can human plasma redox state be used to monitor the progression of rheumatoid arthritis?. ANTIOXIDANTS \& REDOX SIGNALING. 2005;7(9-10):1405-1408.

4. Ueda T, Kamizaki H. Clinical study on correlation with the 60-min plasma phenolsulfonphthalein concentration and other renal function tests. Urol Int. 1978;33(6):435439.

5. Mori A. An antiepileptic drug and cerebroprotection action - Be related with a free radical in particular. Brain 21 . 2001;4(3):293-298.

6. Nejm MB, Haidar AA, Marques MJ, Hirata AE, Nogueira FN, Cavalheiro EA, Scorza FA, et al. Fish oil provides protection against the oxidative stress in pilocarpine model of epilepsy. Metab Brain Dis. 2015;30(4):903-909.

7. Menon B, Ramalingam K, Kumar RV. Oxidative stress in patients with epilepsy is independent of antiepileptic drugs. Seizure. 2012;21(10):780-784.

8. Imazato T, Ueki Y, Hirakata N, Kuroda N, Kishikawa N, Yano M, Shiba T, et al. Oxidation stress degree of the rheumatoid arthritis (RA) patient:About introduction of Ruler "GAP ratio" and the application-Approach of "Sampled Studies". Journal of Analytical Bio-Science.
2012;35(3):225-233.

9. Kitamura K, Nakamura K, Nishiwaki T, Ueno K, Nakazawa A, Hasegawa M. Determination of whether the association between serum albumin and activities of daily living in frail elderly people is causal. Environ Health Prev Med. 2012;17(2):164-168.

10. Morimoto M, Hashimoto T, Suzaki I, Shigeko S, Shimakawa S, Nakatsu T, Kyotani S, et al. Investigation of factors contributing to the current status of patients with severe motor and intellectual disabilities and the relationships between those factors. Journal of severe motor and intellectual disabilities. 2014;39(3):387-395.

11. Fukui T, Yamauchi K, Maruyama M, Yasuda T, Kohno M, Abe Y. Significance of measuring oxidative stress in lifestyle-related diseases from the viewpoint of correlation between d-ROMs and BAP in Japanese subjects. Hypertens Res. 2011;34(9):1041-1045.

12. Kuramoto N, Kitagawa M. Oxidative stress and antioxidant capacity in the postpartum period. J Jpn Acd Midwif. 2012;26(2):201-210.

13. Matsui K, Iwamoto H, Ohtsuki N, Kobayashi T, Miyake $\mathrm{S}$, Yamada M. [The problems of valproate therapy in severely handicapped children - valproate induced hyperammonemia and hypocarnitinemia]. No To Hattatsu. 1991;23(1):32-38.

14. Ohtaki U, Ozawa H, Ishizuka T, Kamiishi A, Sasaki K, Nakajima S, Katayama A, et al. [Evaluation of serum total carnitine values in persons with severe motor and intellectual disabilities with enteral (tube) feeding]. No To Hattatsu. 2012;44(5):374-377.

15. Cao Y, Qu HJ, Li P, Wang CB, Wang LX, Han ZW. Single dose administration of L-carnitine improves antioxidant activities in healthy subjects. Tohoku J Exp Med. 2011;224(3):209-213. 